\title{
Searching for Type Ia Supernovae in Globular Clusters
}

\author{
Rasmus Voss \\ Department of Astrophysics/IMAPP, Radboud University Nijmegen, PO Box 9010, NL-6500 \\ GL Nijmegen, the Netherlands \\ email: rvoss@science.ru.nl
}

\begin{abstract}
Globular clusters are known to have enhanced populations of binaries with compact objects, owing to the dynamical interactions taking place in their dense stellar environments. Both the single degenerate and double degenerate Type Ia supernova progenitors are expected to be similarly enhanced with respect to the field, but the theoretical predictions are highly uncertain. Here we discuss an archival survey aimed at constraining the fraction of Type Ia supernovae that explode inside globular clusters.
\end{abstract}

Keywords. supernovae: general — white dwarfs — galaxies: star clusters

\section{Introduction}

Globular clusters (GCs) are known to harbor large populations of compact binaries. Most well known are the bright low-mass X-ray binaries, and the population of millisecond pulsars and blue stragglers. Recent observational evidence shows that also the populations of white dwarf systems are enhanced (e.g. Dieball et al. 2007; Maccarone \& Knigge 2007; Knigge et al. 2008). The enhancement of tight binaries in globular clusters is a consequence of the very high stellar densities found there (up to $10^{6} M_{\odot} \mathrm{pc}^{-3}$ ). With such high densities, dynamical encounters which create or modify binaries are frequent, and the higher mass of the binaries makes them sink to the center, where the encounter rates are highest.

It is therefore expected that both single degenerate (SD) and double degenerate (DD) progenitors of Type Ia supernovae (SNe Ia) are enhanced in globular clusters. However, it is not well understood which systems lead to supernova explosions and how these systems evolve outside globular clusters, and there are large uncertainties in modeling and observing compact binary populations in globular clusters.

The total fraction of systems found in globular clusters, versus the field depends on the enhancement and on the total mass of globular clusters. The Milky Way has a low abundance of globular clusters, with a combined stellar mass of $\sim 0.1 \%$ of the total stellar mass (e.g. the catalog of Harris 1996). This is in stark contrast to many elliptical galaxies with rich globular cluster systems. Averaging over the population of nearby galaxies yields a likely fraction of SNe Ia in globular clusters of a few per cent (Pfahl et al. 2009).

The most massive globular clusters, with the highest collision rates, are bright and can therefore be seen to large distances $(\sim 50-100 \mathrm{Mpc})$. It is therefore feasible to put limits on the fraction of Type Ia supernovae that explode in globular clusters by identifying these in deep pre- or post-explosion images of the supernova positions (Pfahl et al. 2009). Despite this prediction, no such observational survey has been carried out. 
We are currently performing such a survey using archival observations and literature data to place the first observational constraints on the fraction of SNe Ia in globular clusters.

\section{Archival Survey}

The observations of globular clusters at the positions of type Ia supernovae can be performed before the explosions, but also after, since the light of the globular clusters is provided by stars, which are not affected by the explosion. As the luminosity of the $\mathrm{SNe}$ Ia are very high just after the explosion, it is necessary to wait until they have become fainter than a globular cluster.

Our archival survey is divided into two parts: a literature survey and an observational survey using archival HST observations.

\subsection{Literature Survey}

To facilitate comparisons and thereby the use of Type Ia supernova as standard candles in cosmology, their light curves are observed using BVRI photometry. While most studies are limited to less than $\sim 100$ days, a number of SNe Ia have published late-time data. We have surveyed the literature to compile a sample of 19 such SNe Ia. None of the published results show signs of a constant component expected from a host cluster. We use these to find the intrinsic absolute magnitude of the faintest observation in each band and compare these to the luminosities of globular clusters.

We have also compiled a second more heterogeneous literature sample. This consists of old SNe Ia that were observed before the definition of the BVRI photometry and SNe Ia for which upper limits before the supernova explosions have been published.

\subsection{HST Observations}

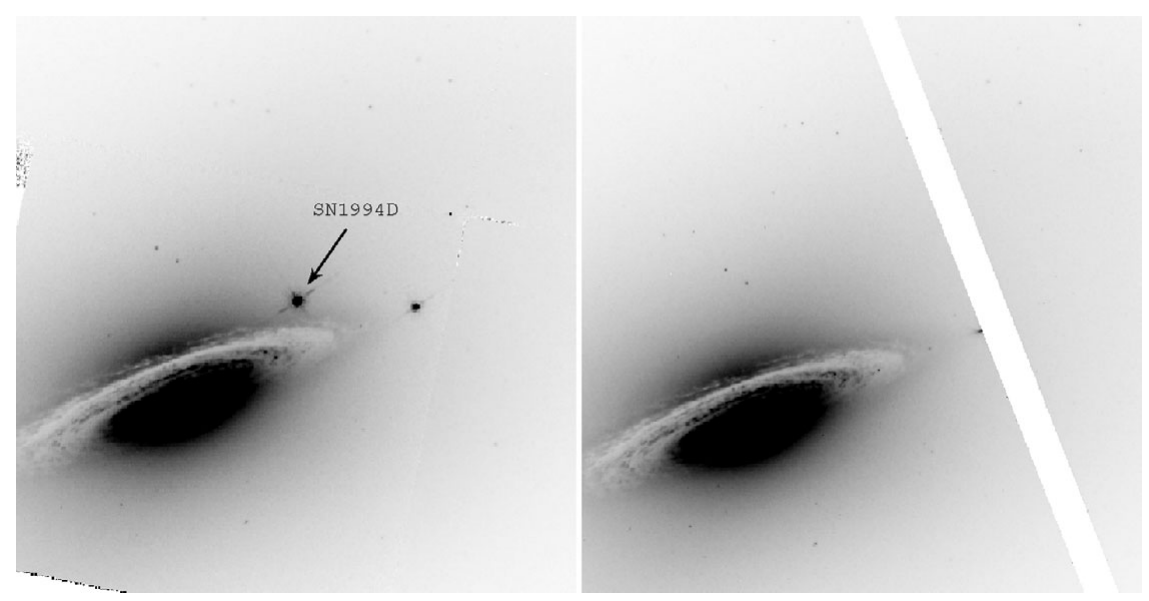

Figure 1. SN1994D in the S0 galaxy NGC 4526 shortly after the explosion (left, HST WFPC2 image using the $F 814 W$ filter) and 9 years after (right, HST ACS WFC image using the F850LP filter). From the right image it can be excluded that the supernova exploded in a massive globular cluster.

\section{Overview}

In addition to the literature survey above, we have searched for archival HST data at the positions of all known SNe Ia within $100 \mathrm{Mpc}$. We analyzed the data to either find 
observations where the supernova is faint enough to exclude a GC origin, or where the supernova was not observed and an interesting upper limit could be inferred. The main problem is the positional accuracy of the sources, as the fields of external galaxies can be crowded. Therefore positions of sub-arcsec precision are needed. Unfortunately many supernova positions are relatively poorly determined, as the only published coordinates are from the original detections with small telescopes, when the supernovae are very bright, and the coordinates are provided without error estimates.

In a few cases, the limits were obtained from images, where the supernova is still seen. In some cases, the positions could be found from other HST images taken when the supernova was visible, which were then matched to the image from which the limit was determined. In the cases where the positions could not be determined from HST images, we used the positions listed in Hicken et al. (2009), who performed careful astrometry of a large sample of supernovae.

\section{Results and Conclusions}

We have not found evidence for a globular cluster origin for any of the Type Ia supernovae in our survey. We have identified 27 Type Ia supernovae for which a globular cluster origin can be completely or partially excluded. The fraction of Type Ia supernovae that explodes in globular clusters is expected to be about a few per cent, but could possibly be significantly higher or lower. The upper limit that can be derived from our sample is similar to, but slightly higher than, this expectation, and excludes theoretically possible models with high fractions. Our results show that a dedicated observational survey will be able to probe the currently favored theoretical predictions.

\section{References}

Dieball, A., Knigge, C., Zurek, D. R., et al. 2007, ApJ, 670, 379

Harris, W. E. 1996, AJ, 112, 1487

Hicken, M., Challis, P., Jha, S., et al. 2009, ApJ, 700, 331

Knigge, C., Dieball, A., Maíz Apellániz, J., et al. 2008, ApJ, 683, 1006

Maccarone, T. \& Knigge, C. 2007, Astronomy and Geophysics, 48, 050000

Pfahl, E., Scannapieco, E., \& Bildsten, L. 2009, Ap. Lett., 695, L111 